\title{
Alzheimer's disease comorbidity in normal pressure hydrocephalus: prevalence and shunt response
}

\author{
J Golomb, J Wisoff, D C Miller, I Boksay, A Kluger, H Weiner, J Salton, W Graves
}

Silberstein Aging and Dementia Research Center, New York University, University School of Medicine, New York, NY 10016, USA

Department of

Neurology

J Golomb

W Graves

Department of

Neurosurgery

J Wisoff

$\mathrm{H}$ Weiner

Department of

Neuropathology

D C Miller

Department of

Psychiatry

I Boksay

A Kluger

J Salton

Correspondence to:

Dr James Golomb

james.golomb@med.nyu.edu

Received 26 August 1999 and in revised form

17 January 2000

Accepted 21 January 2000

\begin{abstract}
The clinical impact of Alzheimer's disease pathology at biopsy was investigated in 56 cognitively impaired patients undergoing shunt surgery for idiopathic normal pressure hydrocephalus (NPH). Cognition was measured by means of the global deterioration scale (GDS), the mini mental status examination (MMSE) and a battery of six psychometric tests. Gait was assessed using objective measurements of velocity and the ambulatory index (AI). The prevalence of cases exhibiting neuritic plaques (positive biopsies) increased in parallel with dementia severity from $18 \%$ for patients with GDS 3 to $75 \%$ for patients with GDS scores $\geqslant 6$. Patients with positive biopsies were more cognitively impaired (higher GDS and lower MMSE scores) as well as more gait impaired (higher AI scores and slower velocities) than patients with negative biopsies. After surgery, gait velocity and AI scores improved significantly and to a comparable degree for patients with and without positive biopsies. Similar proportions of positive and negative biopsy patients also had improved gait as assessed by means of subjective video tape comparisons. There were no significant differences between the biopsy groups in the magnitude of postoperative psychometric change or in the proportion of cases exhibiting improved urinary control. Alzheimer's disease pathology is a common source of comorbidity in older patients with idiopathic NPH where it contributes to the clinical impairment associated with this disorder. For patients accurately diagnosed with NPH, concomitant Alzheimer's disease pathology does not strongly influence the clinical response to shunt surgery. (F Neurol Neurosurg Psychiatry 2000;68:778-781)
\end{abstract}

Keywords: normal pressure hydrocephalus; Alzheimer's disease; dementia; ventriculo-peritoneal shunt

Although normal pressure hydrocephalus $(\mathrm{NPH})$ is recognised as a potentially reversible cause of intellectual and motor impairment in older adults, ventricular shunting procedures do not always result in clinical improvement.
The explanation for shunt unresponsiveness is not well understood but comorbid neurodegenerative pathology such as Alzheimer's disease may play an important part.

Alzheimer's disease is a common cause of dementia in elderly people and may therefore be a frequent source of concomitant pathology in older cognitively impaired patients with NPH. In two recent retrospective studies, clinical improvement was reported in three of five ${ }^{1}$ and two of eight ${ }^{2}$ shunted patients with Alzheimer's disease pathology established by biopsy indicating that comorbidity with this disease does not always mitigate against a beneficial neurosurgical result. Nevertheless, Alzheimer's disease may attenuate the brain's capacity to recover from the effects of NPH and may contribute to many of its cardinal symptoms including dementia, incontinence, and gait impairment. ${ }^{3}$ The extent to which Alzheimer's disease pathology may negatively influence the response to shunt surgery requires prospective investigation using quantitative clinical assessment techniques.

In this study we estimated the prevalence of Alzheimer's disease pathology in 56 cognitively impaired patients who received ventriculoperitoneal shunts for a diagnosis of idiopathic NPH. The presence of pathology was ascertained by means of cortical biopsies obtained during surgery. The impact of Alzheimer's disease on the clinical presentation of the patients and on their response to shunting was assessed through psychometric and quantitative gait evaluations performed at baseline and postoperatively.

\section{Methods}

Within a 55 month period, 117 patients at our institution received shunt operations for a diagnosis of idiopathic NPH. All patients evidenced prominent signs of dyspraxic gait instability and MRI or CT disclosing severe ventricular enlargement in excess of sulcal prominence as interpreted by a neuroradiologist unaware of the patient's clinical condition. These patients received a diagnosis of NPH because neurological and medical evaluations disclosed no alternative explanation for the gait dysfunction. Specifically, in no patient could the gait impairment be attributed to rheumato- 
logical disease, peripheral neuropathy, myelopathy, stroke, Binswanger's disease, Parkinson's disease, cerebellar disease, or intracranial mass. Of the 117 patients, 81 underwent a structured psychiatric interview preoperatively and 77 were found to be cognitively impaired (global deterioration scale ${ }^{4}$ (GDS) score of $\geqslant 3$ ). In all cases, cognitive dysfunction either followed or evolved simultaneously with the onset of a gait disorder. After informed consent, 56 of the 77 cognitively impaired patients received cortical biopsies during shunt surgery. Biopsies were obtained when patients, their families, or their referring physicians sought information on the presence of comorbid neurodegenerative disease. The biopsy consisted of a $5 \mathrm{~mm} \times 2 \mathrm{~mm}$ wedge of tissue through the thickness of the cortex obtained adjacent to the shunt penetration site. Shunts were installed right frontally in 18 patients and right occipitoparietally in 38 patients.

The biopsy specimens were fixed in $10 \%$ neutral buffered formalin and processed routinely into paraffin for histological examination. Sections were examined with haematoxylin and eosin stains, with Congo red stains, and with Bielschowsky silver stains. The biopsies were evaluated for inflammation, neoplastic cells, amyloid angiopathy, neuritic and diffuse plaques, neurofibrillary tangles, Lewy bodies, and other intracellular inclusions. Biopsies were considered positive for Alzheimer's disease pathology if neuritic plaques, in any number, were present on the Bielschowsky stained sections.

Cognition was assessed by means of five psychometric tests previously found sensitive to shunt related improvement in a wider sample of patients with idiopathic NPH. The tests, which included measures of verbal recall, perceptual processing, psychomotor praxis and motor planning consisted of the Guild paragraph recall test, ${ }^{5}$ the digit symbol substitution test, ${ }^{6}$ the trail making test, ${ }^{7}$ the Mefferd and Moran perceptual speed test, ${ }^{8}$ and the Purdue pegboard test. ${ }^{9}$ to facilitate statistical analysis and data presentation, a composite cognitive score equal to the mean of the standardised ( $\mathrm{Z}$ transformed) raw scores was calculated. Gait was evaluated by measuring average velocity over a 60 foot distance. Additionally, by viewing pairs of digitised video clips made preoperatively and postoperatively, two clinicians independently recorded subjective impressions of gait change using a seven point ordinal scale: $(+3=$ dramatic improvement/ resolution of deficit, $+2=$ definite/moderate improvement, $+1=$ questionable/mild improvement, $0=$ no change, $-1=$ questionable $/ \mathrm{mild}$ deterioration, $-2=$ definite $/$ moderate deterioration, $-3=$ marked deterioration). The raters had no previous clinical involvement in the study and were blinded to both the biopsy result as well as to the presurgical/postsurgical identity of the individual clips. A mean of the two rater's scores was computed and a value of $\geqslant+2$ was conservatively taken as evidence for postshunt improvement. Finally the ambulatory index ${ }^{10}$ (AI), a six point ordinal scale assessing functional gait capacity, was administered. Baseline velocity and AI scores were available on 43 and 42 patients respectively and 35 completed the psychometric battery. A subset of these patients returned for postoperative clinical testing (mean follow up $=4.3$ months). A complete set of preoperative and postoperative psychometric results was available for 27 patients, velocity measurments for 32 , clinician's impression of gait change (CIGC) ratings for 33, and AI scores for 34. There were no significant GDS, MMSE, sex, or age differences at baseline between patients with and without postoperative follow up data.

\section{Results}

No biopsy showed inflammation, neoplasm, neurons with Lewy Bodies, Pick bodies, or glial cells with silver positive inclusions. Amyloid angiopathy and neuropil threads were detected in only a few specimens and sparse accumulations of neurofibrillary tangles were seen in six. Neuritic plaques were found in 23 biopsies

\begin{tabular}{|c|c|c|c|c|}
\hline & \multirow[b]{2}{*}{$n(A D-/ A D+)$} & \multicolumn{2}{|l|}{ Biopsy group } & \multirow{2}{*}{$\begin{array}{l}\text { p Value } \\
\text { between group } \\
\text { contrast }\end{array}$} \\
\hline & & $A D-$ & $A D+$ & \\
\hline \multicolumn{5}{|l|}{ Clinical characteristics at baseline: } \\
\hline Age (mean (SD)) & $33 / 23$ & $76.15(7.65)$ & $77.56(6.54)$ & 0.474 \\
\hline Male/female ratio & $33 / 23$ & $21 / 12$ & $12 / 11$ & 0.391 \\
\hline GDS (mean (SD)) & $33 / 23$ & $4.03(0.85)$ & $4.91(1.08)$ & 0.001 \\
\hline MMSE (mean (SD)) & $33 / 23$ & $22.55(4.84)$ & $15.91(8.24)$ & 0.001 \\
\hline Composite cognitive score (mean (SD)) & $21 / 14$ & $0.16(0.74)$ & $-0.62(0.72)$ & 0.004 \\
\hline Gait velocity $(\mathrm{cm} / \mathrm{s}$; mean $(\mathrm{SD}))$ & $27 / 16$ & $71.14(25.65)$ & $49.14(34.12)$ & 0.021 \\
\hline Ambulatory index (mean (SD)) & $26 / 16$ & $3.15(1.43)$ & $4.22(1.66)$ & 0.034 \\
\hline Patients with incontinence $(\%)$ & $33 / 23$ & 73 & 74 & 0.921 \\
\hline \multicolumn{5}{|l|}{ Shunt response: } \\
\hline \multicolumn{5}{|l|}{ Change in cognition } \\
\hline GDS (mean change (SD)) & $21 / 16$ & $0.05(0.86)$ & $0.00(0.63)$ & 0.854 \\
\hline MMSE (change (SD)) & $24 / 16$ & $-0.46(2.72)$ & $0.88(5.26)$ & 0.516 \\
\hline $\begin{array}{l}\text { Composite cognitive score (mean change in } \\
\mathrm{Z} \text { score (SD)) }\end{array}$ & $17 / 10$ & $0.29(0.32)^{\star \star}$ & $0.23(0.34) \dagger$ & 0.653 \\
\hline \multicolumn{5}{|l|}{ Change in gait } \\
\hline Velocity (mean change; cm/s (SD)) & $19 / 13$ & $17.36(18.12)^{\star \star}$ & $20.74(24.68)^{\star}$ & 0.658 \\
\hline $\begin{array}{l}\text { CIGC (\% exhibiting moderate or marked } \\
\text { improvement) }\end{array}$ & $21 / 12$ & 42.8 & 41.7 & 0.947 \\
\hline Ambulatory index (mean change (SD)) & $20 / 14$ & $0.65(1.14)^{\star}$ & $0.86(1.46)^{\star}$ & 0.645 \\
\hline \multicolumn{5}{|l|}{ Change in incontinence } \\
\hline Incontinent cases improved (\%) & $20 / 14$ & 65.0 & 57.1 & 0.643 \\
\hline
\end{tabular}


whereas 12 patients showed only diffuse plaques. The plaque density by CERAD $^{11}$ criteria was sparse in 10 and moderate to frequent in the remaining 13. Applying the recommended age adjustments, a CERAD diagnosis of definite Alzheimer's disease could be made in seven cases, probable disease in nine, and possible disease in seven.

The clinical characteristics at baseline for the biopsied patients are presented in the first section of the table. Compared with Alzheimer's disease negative patients, those with Alzheimer's disease pathology were more cognitively impaired as evidenced by significantly higher GDS and lower mini mental status examination (MMSE) scores. The Alzheimer's disease positive group also exhibited greater gait dysfunction as exemplified by slower velocities and higher ambulatory index scores. There were, however, no significant between group differences for age, male/female ratio, frequency of incontinence, biopsy site, or surgical complications. The figure depicts the prevalence of Alzheimer's disease pathology as a function of GDS level. Whereas only $18 \%$ of GDS 3 patients were Alzheimer's disease positive, the prevalence increased to $75 \%$ for GDS 6 and above.

The shunt response data for the subset of patients who returned for postoperative testing is summarised in the second section of the table. Relative to their preoperative baselines, both Alzheimer's disease positive and Alzheimer's disease negative patients exhibited statistically significant improvements in gait velocity and AI scores. Comparable gait improvement was also recorded on the CIGC and similar proportions of patients experienced improved urinary control. For the Alzheimer's disease negative group, a small but statistically significant gain of $0.29 \mathrm{SD}$ was found on the composite cognitive score whereas a trend $(p<0.06)$ for improvement was seen for the Alzheimer's disease positive patients. Neither MMSE nor GDS scores improved significantly with shunting. No significant biopsy result by shunt outcome interactions were detected for any of the clinical measures. Within the Alzheimer's disease positive patients, no significant correlation was found between neuritic plaque density as estimated using the CERAD

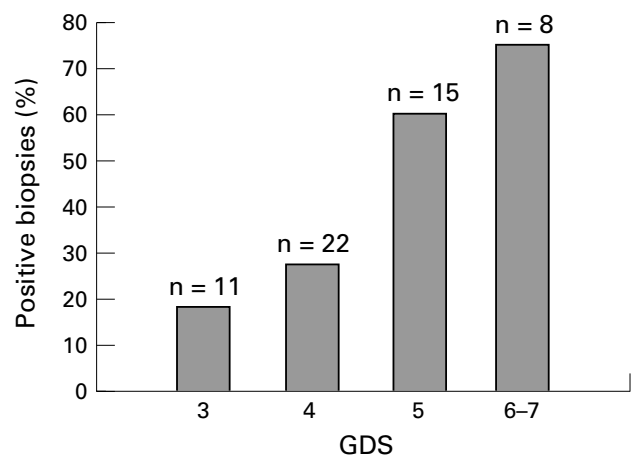

Percentage of biopsies evidencing neuritic plaques as a function of GDS score. A GDS score of 3 indicates mild cognitive impairment while scores of 4 through 7 indicate progressively severe levels of dementia. criteria ${ }^{11}$ and change on any of the measured clinical indices.

\section{Discussion}

This study shows Alzheimer's disease pathology to be common in patients with NPH where its prevalence increases in proportion to the severity of dementia. These results are consistent with previous findings that hippocampal atrophy, a characteristic of Alzheimer's disease, correlates with cognitive performance in elderly patients with $\mathrm{NPH}^{12}$ and suggests that Alzheimer's disease may contribute significantly to the dementia of many patients diagnosed with this disorder. Although Alzheimer's disease positive patients with NPH were more cognitively impaired and exhibited a disproportionate level of gait dysfunction, their improvement after shunt placement was comparable with patients with negative biopsies.

Although our biopsy based prevalence estimates are roughly consistent with those reported elsewhere, ${ }^{12}{ }^{13}$ very small neocortical tissue samples may be an inexact means of establishing a pathological diagnosis of Alzheimer's disease. Alzheimer's disease pathology typically affects neocortical regions only after involvement of the medial temporal lobe ${ }^{14}$ and sampling error may have led us to underestimate the true frequency with which the disease complicates the diagnosis of NPH. On the other hand, because low levels of neuritic plaques are traditionally held compatible with "normal aging", we also may have overestimated the prevalence of Alzheimer's disease in our sample. Emerging evidence that the appearance of neocortical neuritic plaques tends to coincide with the development of cognitive impairment implies that previously accepted thresholds for establishing a diagnosis of Alzheimer's disease may have been too restrictive. ${ }^{15}$ In view of these considerations, we suspect that the true prevalence of Alzheimer's disease is probably greater than our reported data suggest. Nevertheless, although we accepted any neuritic plaques as evidence for the presence of Alzheimer's disease pathology, we remain neutral with respect to the minimum plaque density required to establish a pathological diagnosis.

Patients with NPH and Alzheimer's disease would be expected to progressively worsen with the passage of time but shunt surgery may lessen their overall burden of functional disability and slow what would otherwise be an accelerated trajectory of neurological decline. Longitudinal follow up studies are needed to investigate the long term benefit of shunt operations in Alzheimer's disease positive patients with NPH.

A decision to recommend shunt surgery is often difficult and physicians must carefully weigh the potential for complications against an often uncertain likelihood of postoperative improvement. The potential availability of specific biological and neuroimaging tests for increasing confidence in the diagnosis of Alzheimer's disease will introduce an additional level of complexity to the decision making process. Our present findings suggest that 
patients with NPH who are good candidates for shunt operations based on accepted clinical and radiographic criteria should not be denied surgery solely because Alzheimer's disease may be suspected as a concomitant diagnosis.

1 Del Bigio MR, Cardoso ER, Halliday WC. Neuropathological changes in chronic adult hydrocephalus: cortical biopsies and autopsy findings. Can f Neurol Sci 1997; 24:121-6.

2 Savolinen S, Paljarvi L, Vapalahti M. Prevalence of Alzheimer's disease in patients investigated for presumed Alzheimer's disease in patients investigated for presumed normal pressure hydrocephalus: a clinical and neuropa
logical study. Acta Neurochirurgica 1999;141:849-53.

logical study. Acta Neurochirurgica 1999;141:849-53.
Visser H. Gait and balance in senile dementia of Alzheimer's

3 Visser H. Gait and balance in senile
type. Age Ageing 1983;12:296-301.

type. Age Ageing 1983;12:296-301.
Reisberg B, Ferris SH, de Leon MJ, et al. The global deterioration scale for assessment of primary degenerative dementia. Am f Psychiatry 1982;139:1136-9.

5 Gilbert JG, Levee RF, Catalano FL. A preliminary report on a new memory scale. Percept Mot Skills 1968;27:277-8.

6 Wechsler D. Wechsler adult intelligence scale. New York: Psychological Corporation, 1955.

7 Reitan RM. Validity of the trail making test as an indicator of organic brain damage. Percept Mot Skills 1958;8:271-6.

8 Moran LJ, Mefferd RB. Repetitive psychometric measures.

Psychol Rep 1959;5:269-75.
9 Tiffin J, Asher EF. The Purdue pegboard: norms and studies of reliability and validity. F Appl Psychol 1948;32:234-47.

10 Hauser SL, Dawson DM, Lehrich JR, et al. Intensive immunosuppression in progressive multiple sclerosis. A randomized, three-arm study of high-dose intravenous cyclophosphamide, plasma exchange, and ACTH. N Engl f Med 1983;308:173-80.

11 Mirra SS, Heyman A, McKeel D, et al. The consortium to establish a registry for Alzheimer's disease (CERAD). Part II. Standardization of the neuropathologic assessment of Alzheimer's disease. Neurology 1991;41:479-86.

12 Golomb J, de Leon MJ, George AE, et al. Hippocampal atrophy correlates with severe cognitive impairment in elderly patients with suspected normal pressure hydrocephalus. $\mathcal{F}$ Neurol Neurosurg Psychiatry 1994;57:590-3.

13 Bech RA, Juhler M, Waldemar G, et al. Frontal brain and leptomeningeal biopsy specimens correlated with cerebrospinal fluid outflow resistance and B-wave activity in patients suspected of normal-pressure hydrocephalus. Neurosurgery 1900;40:497-502.

14 Braak H, Braak E, Yilmazer D, et al. Pattern of brain destruction in Parkinson's and Alzheimer's diseases [review]. Fournal of Neural Transmission (Budapest) 1996;103: 455-90.

15 Price JL, Morris JC. Tangles and plaques in nondemented aging and "preclinical" Alzheimer's disease. Ann Neurol 1999;45:358-68.

\section{NEUROLOGICAL STAMP}

\section{August Forel (1848-1931)}

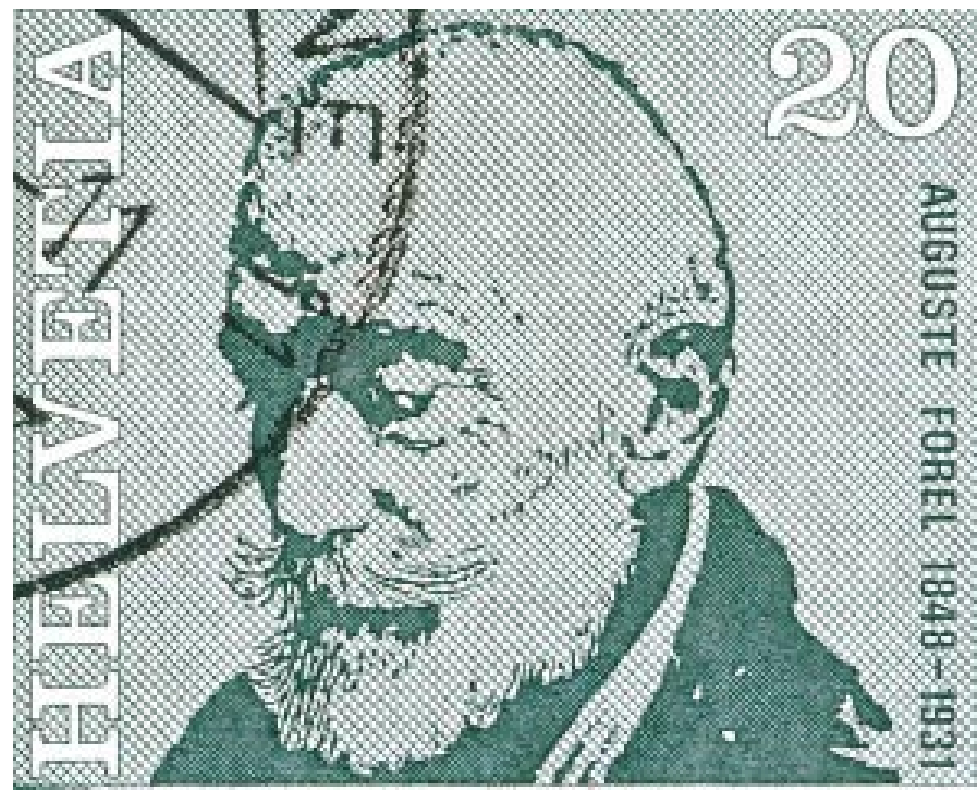

August Forel was Professor of Psychiatry in Zurich. He made the first complete section of the whole brain in 1875 and performed extensive neuroanatomical research. His collected works (1907) included description of the tegmental fields of Forel and the zona incerta. In 1887 he published one of his most important works, on the neuron theory describing cellular functional units within the brain. His investigations also included the anatomy of the thalamus and he described in considerable detail the trigeminal, pneumogastrlc, and hypoglossal nerves and the hypothalamus. One of the areas of the hypothalamus was named the ventral tegmental decussation of Forel.

He was a wine drinker and possibly an alcoholic but recognised the problem and took a total abstinence pledge in 1886. In 1889 he founded an Institute in Zurich for the medical treatment of alcoholism and throughout his career he worked for social reforms to prevent mental illness, syphilis, and alcoholism. Forel was a strong advocate of the League of Nations. As an ardent pacifist he attempted to bring the first war to a close. He was also an entomologist and after retirement in 1893 devoted the rest of his life to social reform and the study of the psychology of ants. He had earlier studied the internal anatomy of ants and proposed a new taxonomy of these insects.

Forel was honoured philatelically by Switzerland in 1971 in a series of stamps honouring famous physicians (Stanley Gibbons 820, Scott 536). 